
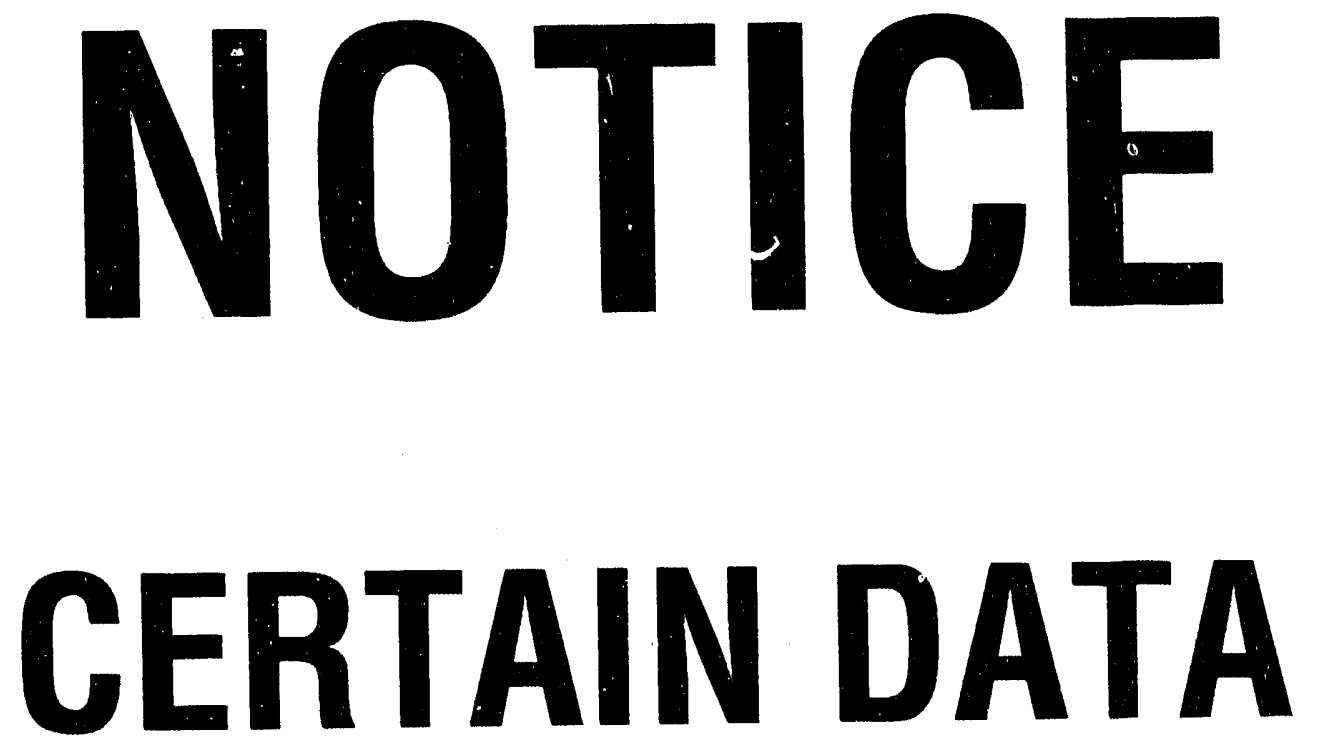

CONTAINED IN THIS
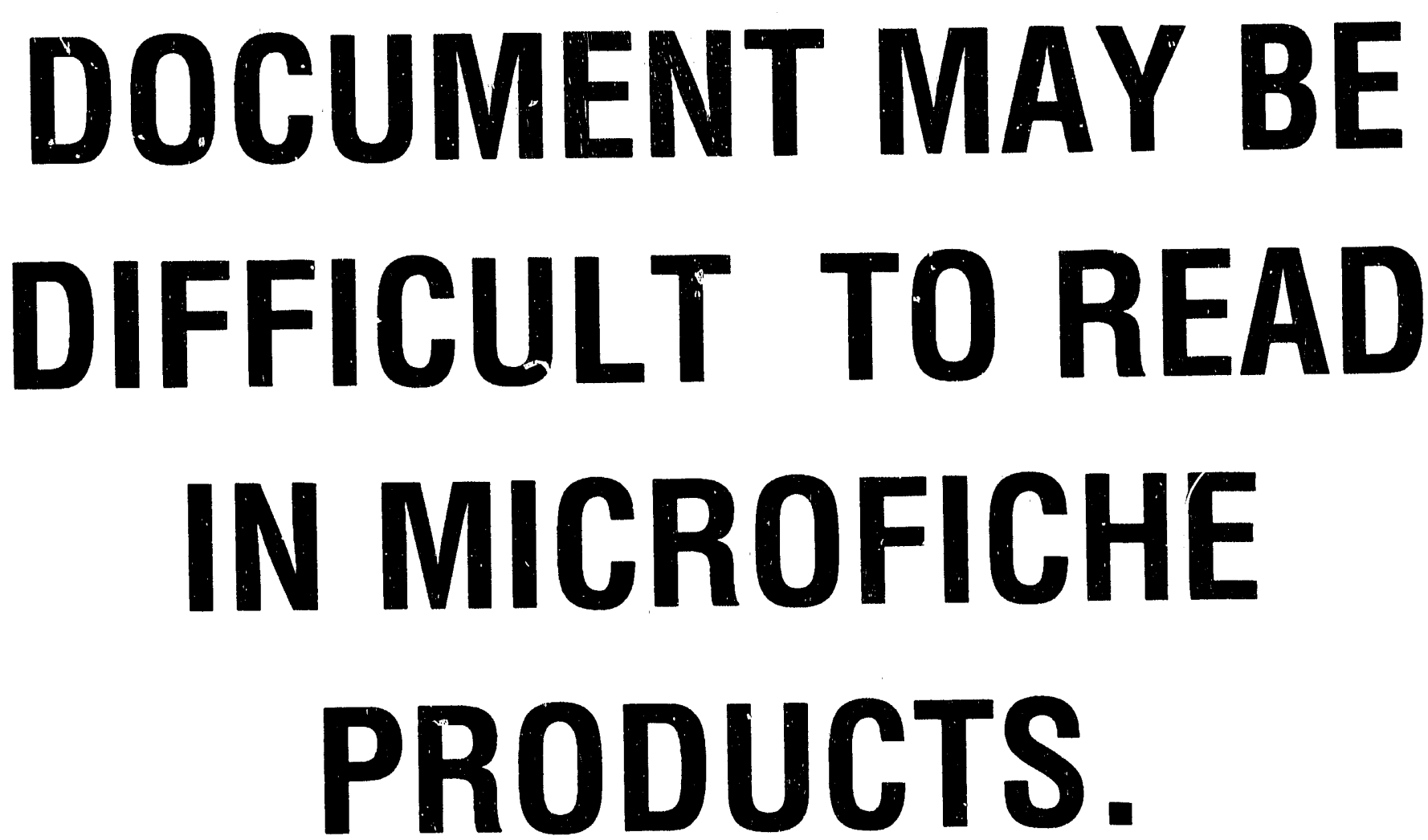


\title{
A HIGH RESOLUTION INTERFEROMETER FOR USE WITH SYNCHROTRON RADIATION
}

\author{
K.D. Moeller \\ Physics Department \\ Fairleigh Dickinson University, \\ Teaneck, NJ 07666 and \\ New Jersey Institute of Technology \\ Newark, NJ 07102 \\ T. Sears \\ Chemistry Department \\ Brookhaven National Laboratory \\ Upton, NY 11973 \\ H.T. Liu \\ University of Science and Technology of China \\ Hefei, Peoples' Republic of China \\ C.J. Hirschmugl and G.P Williams \\ National Synchrotron Light Source \\ Brookhaven National Laboratory \\ Upton, $N Y 11973$
}

\begin{abstract}
We present the first results from a new instrument which is designed to work at very high resolution in the infrared spectral region but whose principles can be used in the vuv/soft-xray region. The instrument is based on an interferometer. For the vuv/softxray region the beamsplitting is achieved by wavefront division which takes advantage of the spatial coherence of synchrotron radiation. Normally the highest frequency in the spectrum from an interferometer is determined hy the sampling frequency, which has to be at least twice that of the highest frequency in the spectrum. We show that this can be avoided if the spectral range is deliberately restricted by a grating or by the use of an undulator. In this case the interferograms contain a known and restricted range of frequencies which lead to an unambiguous assignment during the Fourier processing required to generate the spectrum.
\end{abstract}

\section{WASTR}

-Work performed under the auspices of the U.S. Department of Energy, under contract DE-AC02-76C110016. 2, 


\section{INTRODUCTION}

The development of infrared synchrotron at the NSLS[1,2], has utilized Michel....... Lamellar interferometers for spectral analysis. The latter led to a new instruncint in which the spatial coherence of the infrared synchrotron radiation was utilized by deriving two beams for the interferometer by physically dividing the wavefront emerging from the ring[3].

We now present details and preliminary results from a new instrument which can be operated either using a wavefront dividing beamsplitter or using conventional amplitude dividing beamsplitters.

\section{THE INTERFEROMETER}

(a) Resolution.

The crucial component of the new instrument is shown schematically in Fig. 1 and in the photograph of Fig. 2. The acrual instrument is installed in a large vacuum tank capable of operating at a vacuum of $10^{-2}$ torr. It is called a Mach-Zender interferometer because it has two "beamsplitters" - one for separating the two beams and one for recombining them. It is also a multiple path interferometer in which the central pillar moves, thus shortening the path length on one side while lengthening it on the other. The beam makes a total of 10 passes per side, which means that the maximum path difference is 20 times the maximum travel $(15 \mathrm{~cm})$ of the central column. The instrument can be used in two ways - in one of which the central column travels $\pm 15 \mathrm{~cm}$ to generate double sided interferograms of maximum resolution $\Delta v$ of $1 / 600 \mathrm{~cm}^{-1}$ or $.0017 \mathrm{~cm}^{-1}$. (The resolution of an interferometer is $1 / 2 \mathrm{~L}$ where $\mathrm{L}$ is the maximum path difference[4]). In another mode the zero path difference position can be shifted to the edge of the scan range of the central column by moving one of the end columns out by $30 \mathrm{~cm}$. The maximum path difference then is $600 \mathrm{~cm}$ giving a maximum 
resolution, $\Delta v$, of $1 / 1200 \mathrm{~cm}^{-1}$ or $.0008 \mathrm{~cm}^{-1}$.

(b) Wavelength Range (Highest Measurable Frequency)

An equally important consideration for interferometers and one which assumes significantly more importance in a vuv interferometer is the highest frequency measurable with a given instrument. This is related to the sampling interval while the central pillar is moved. Information theory states that the measuring interval must be at least twice that of the highest frequency present. Since, in this instrument, the path is multplied, the sampling interval is also a multiple of the actual distance traveled by the central column. As an example, if we wish to obtain a spectrum up to $1000 \mathrm{~cm}^{-1}$, then we have to sample the modulation at twice that frequency. Since for $v=1000$ $\mathrm{cm}^{-1}, \lambda=10 \mu$, then we would have to sample every $5 \mu$ of optical path difference, or for each central pillar displacement of $0.25 \mu$. This makes the instrument more challenging from a mechanical engineering point of view but may be obviated in the following way.

If the input spectrum is reduced in width, but if the spectral band is chosen such that its maximum frequency $v_{\text {MAX }}$ is equal to an integer, $f$, times its width, $\Delta v$, then the sampling interval can be chosen to be $f$ times larger[5]. The maximum path difference is the same but fewer data points are taken as one is sampling the sub-harmonics of the actual spectrum. The Fourier transform turns out to be unambiguous, provided the above conditions are met. It is especially important that there be no spectral information between the band being measured and zero frequency.

In order to prepare for this we used a grating spectrometer between the interferometer and the detector. Clearly the angle of the grating could be used to select the center of a band whose width ould be determined by the size of a slit.

\section{DISCLAIMER}

\footnotetext{
This reporn was prepared as an account of work sponsored by an agency of the United States Government. Neither the United States Gruvernment nor any agency thereof, nor any of their employees, makes any warranty, express or implied, or assumes any legal liability or responsibility for the accuracy, completeness, or usefulness of any information, apparatus, product, or process disclosed, or represents that its use would not infringe privately owned rights. Reference herein to any specific commercial product, process, or service by trade name, trademark, manufacturer, or otherwise does not necessarily constitute or imply its endorsement, recommendation, or favoring by the United States Government or any agency thereof. The views and opinions of authors expressed herein do not necessarily state or reflect those of the United States Government or any agency thereof.
} 


\section{RESULTS}

In order to test the instrument we measured the absorption spectrum of ammonifili. The sample was in a meter long gas cell, which was an integral part of the expcimental arrangement lying between the interferometer and the grating. The gas cell had a spherical mirror to refocus the light from the entrance spot to the exit spot. Ammonia was chosen since it has a series of rotational bands, separated from each other by about $20 \mathrm{~cm}^{-1}$ starting at $40 \mathrm{~cm}^{-1}$.

In Fig. 3 we show the spectrum obtained over the region $0-200 \mathrm{~cm}^{-1}$, in which 7 of these bands can clearly be distinguished. We also point out "artifacts" due to the $50 \mu$ mylar beamsplitter at $-65 \mathrm{~cm}^{-1}$, and due to a quartz filter at $-127 \mathrm{~cm}^{-1}$. This spectrum was taken at a resolution of $3.56 \mathrm{~cm}^{-1}$ and using 128 points, at one point per second.

As a verification of the ability to define a spectral bandpass using a grating, (to enable higher frequencies to be measured more easily), we show in Fig. 4 a spectrum of another ammonia sample in the range $0-250 \mathrm{~cm}^{-1}$ in which it can be seen that spectral information is only present in the $10-60 \mathrm{~cm}^{-1}$ region. This region was defined using an echelette grating of 10 lines per mm. blazed at $29^{\circ}\left(\lambda_{B}=42 \mu, 238 \mathrm{~cm}^{-1}\right)$. The resolution of this spectrum was $3.8 \mathrm{~cm}^{-1}$. At these low frequencies it was not necessary to use the sub-harmonic method described earlier to attain higher resolution. We can demonstrate higher resolution, however, by running at a maximum path difference corresponding to a $\delta v$ of $.325 \mathrm{~cm}^{-1}$ and shown in the insert to Fig. 4. In this spectrum we were able clearly to resolve the $J=1$ line at $40 \mathrm{~cm}^{-1}$ into a doublet caused by the inversion of the molecule.

\section{DISCUSSION}

We have built a novel instrument for : 2 e far infrared spectral region in the range 1-400 $\mathrm{cm}^{-1}$ which comprises both an interferometer and a grating. The combination will 
enable high resolution spectra to be obtained at higher frequencies using fewer data points. The instrument operates completely in vacuum and is equipped with a : ... long gas cell. We have demonstrated the performance of the instrument by the absorption of ammonia gas.

\section{ACKNOWLEDGEMENTS}

We are extremely grateful for the technical support provided by Dennis Carlson. This work was supported by the United States Department of Energy under contract DEAC02-76CH0OO16.

\section{REFERENCES}

1. G.P. Williams, Nucl. Instr. \& Methods in Physics Research, A291 (1990) 8.

2. C.J. Hirschmugi, this volume.

3. K.D. Moeller, D.P. Siddons, C.J. Hirschmugl, D. Scardino, P. Petrone, D. Carlson and G.P. Williams, Applied Optics, 30 (1991) 4297.

4. K. D. Moeller and W.G. Rothschild, "Far-Infrared Spectroscopy", Wiley, New York (1971) 139.

5. J. Kachmarsky, C. Belorgeot, A. Pluchino and K.D. Moeller, Applied Optics 15 (1976) 708 .

6. J.M. Dowling, J. Mol. Spectroscopy, 27 (1968) 527. 


\section{FIGURE CAPTIONS}

Fig. 1. Schematic of the Mach-Z̈ender interferometer. The light makes 10 passid either side of the central column, which moves to change the path lengths.

Fig. 2. Photograph of the interferometer section shown in Fig. 1, which is at the heart of the present instrument.

Fig. 3. Th: pure rotational spectrum of ammonia gas as measured by the U4IR MachZender interferometer. 7 lines are seen and labeled. In addition two features appear as artifacts due to the quartz filter and to the beamsplitter.

Fig. 4. The spectrum obtained by a combination of the interferometer and the grating. The latter defines the more limited bandpass seen. In the inset is seen a narrow region measured at higher resolution and in which the inversion splitting of the first rotational band can be seen. 


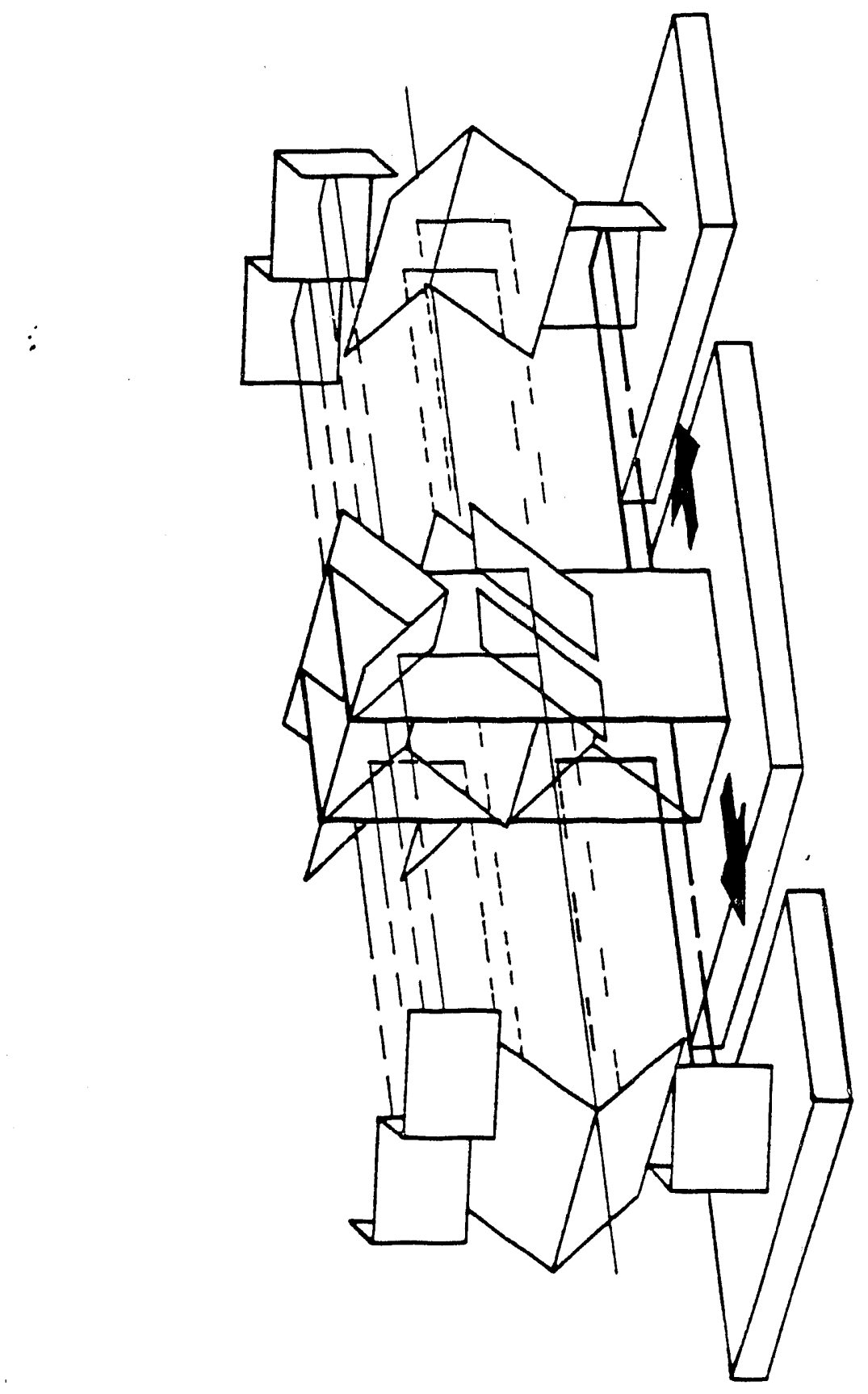




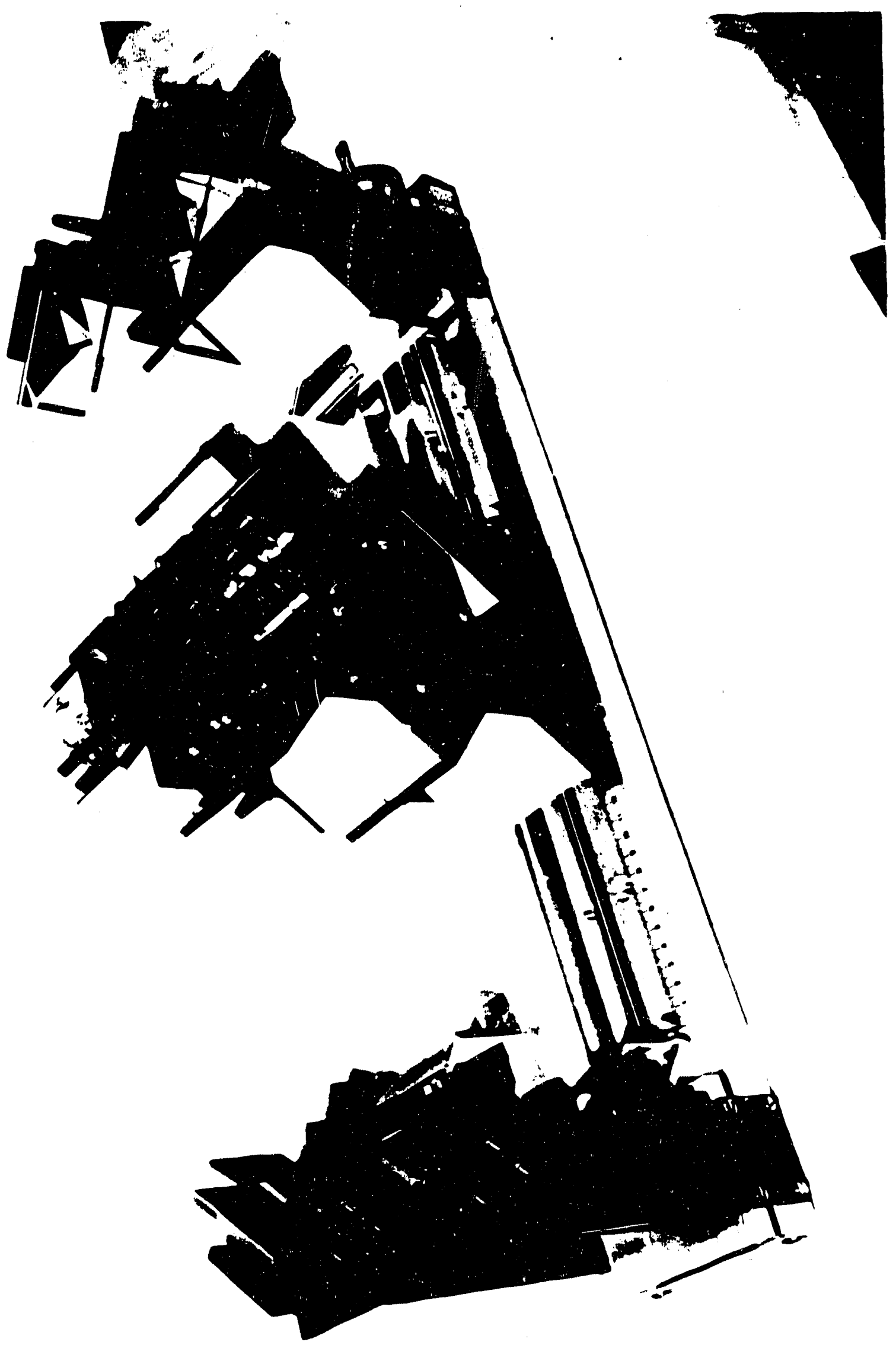




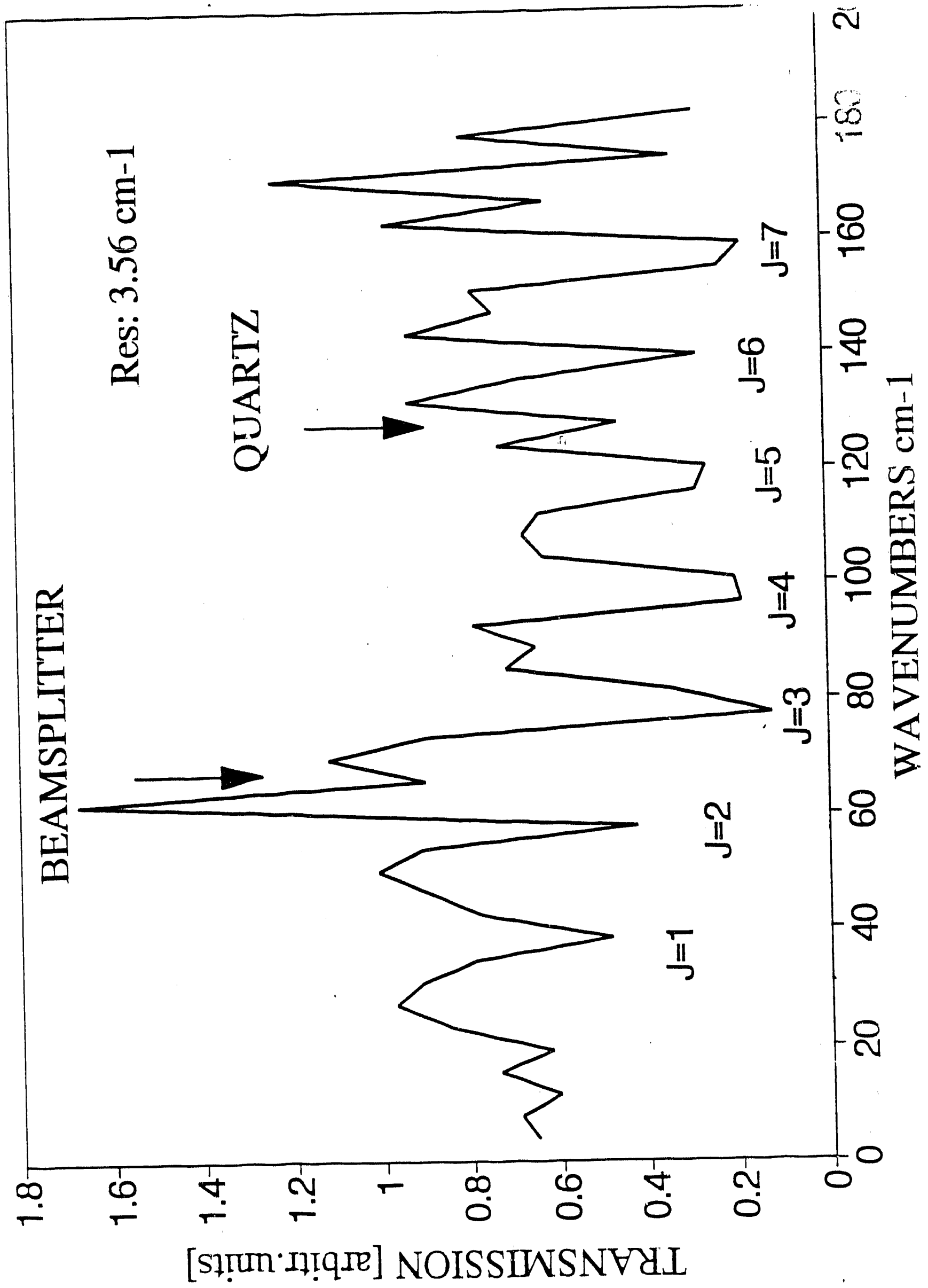




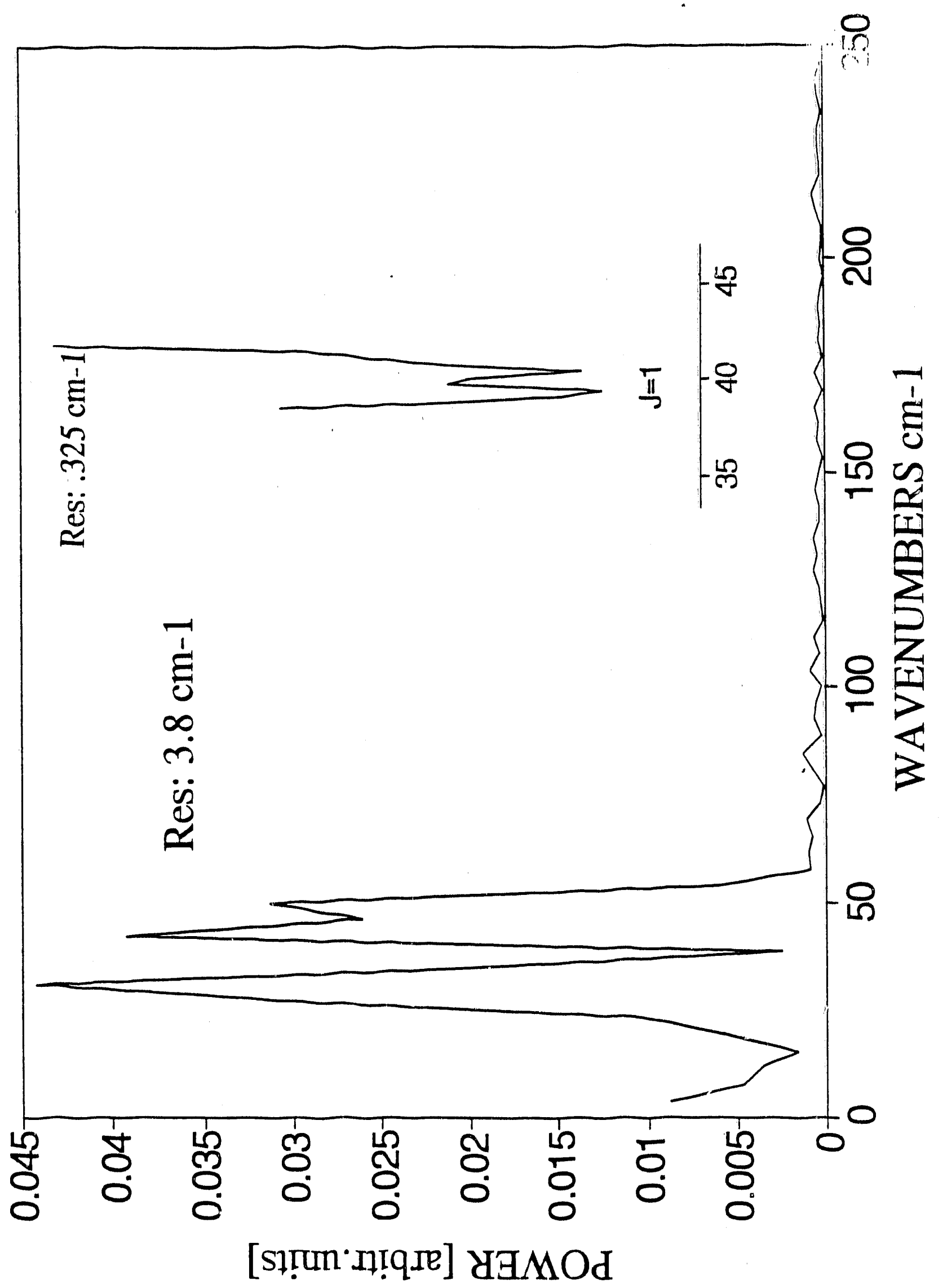



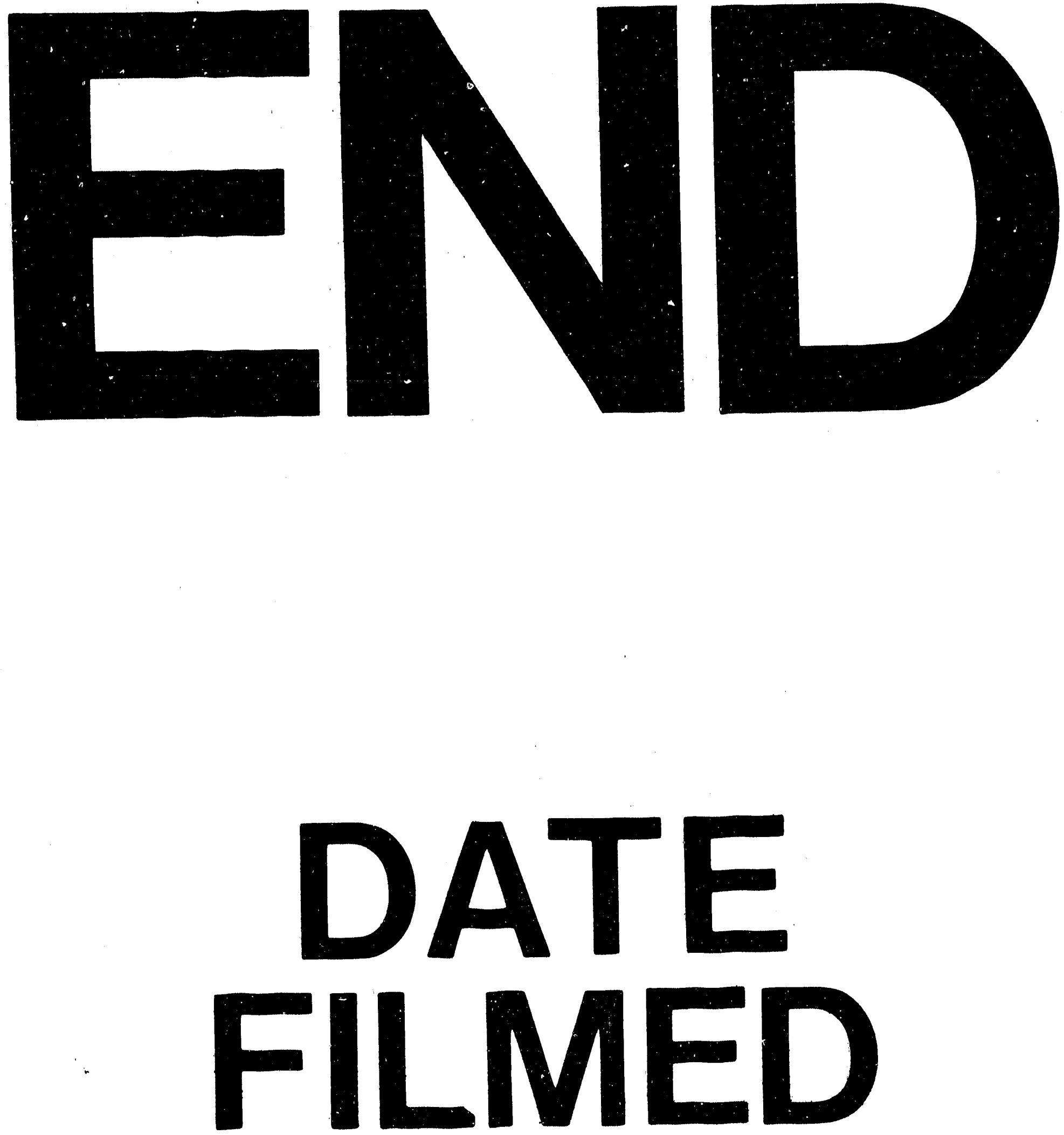

1

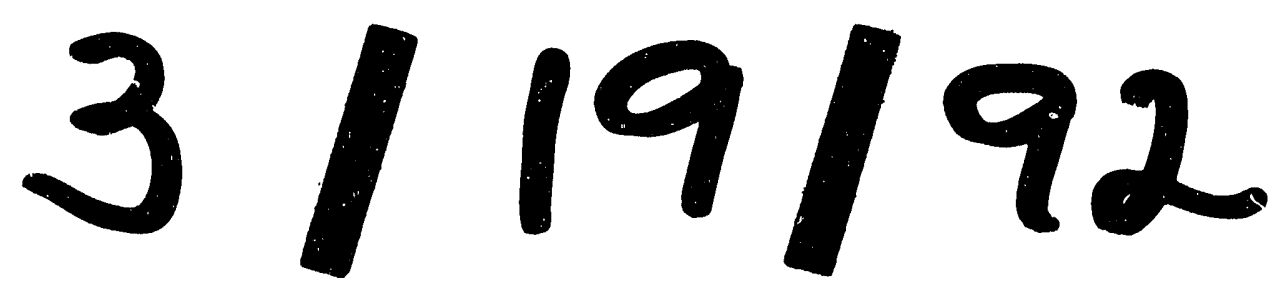


